\title{
PERCEPCIÓN DEL ORIENTADOR ACERCA DE LA IMPORTANCIA QUE ATRIBUYE LA COMUNIDAD EDUCATIVA A SUS FUNCIONES
}

\author{
Jacobo Cano Escoriaza \\ Universidad de Zaragoza \\ Narciso García Nieto \\ Universidad Complutense de Madrid
}

RESUMEN: El presente trabajo analiza las percepciones que tienen los orientadores que llevan a cabo su tarea en los diferentes centros que imparten la ESO en Aragón. Se pone de manifiesto la valoración que la comunidad educativa hace de su trabajo profesional, así como la distinta importancia a sus funciones como orientadores. Resulta relevante desarrollar estrategias que favorezcan el trabajo colaborativo para mejorar la calidad educativa. Las múltiples funciones y tareas que se les piden a los orientadores escolares son tan variadas y complejas que merecen ser analizadas y priorizadas porque no todas tienen la misma importancia y alcances. Se corre el riesgo de confundir las que son urgentes con las que son importantes. Conocer las percepciones que la comunidad educativa -profesorado, padres, tutores y alumnado- tienen sobre ellas puede ser una fuente de conocimiento de la realidad para poder transformarla.

ABSTRACT: This paper analyzes the perceptions of school counsellors working in Secondary Schools in the region of Aragon. It studies the assessment of the counsellors' work by the school community. and the different importance given to their tasks as counsellors. It is important to develop strategies to promote collaborative working to improve the quality of the education. The different tasks assigned to school counsellors are so assorted and complex that they have to be carefully analyzed and assessed, because each one has its significance and outreach. We run the risk of mixing the urgent ones with the important ones. To know the perceptions on these tasks by the school community -teachers, parents, tutors, and students- can be very enlightening to make any needed changes.

PALABRAS CLAVE: Atribución de funciones, comunidad educativa, orientación escolar, orientador, percepción del rol.

KEYWORDS: Function assignment, School Conimunity, School counselling, Counsellor, Role's perception. 


\section{INTRODUCCIÓN: APROXIMACIÓN AL ESTADO DE LA CUESTIÓN}

Las funciones de los orientadores escolares en el ámbito de la Educación Secundaria Obligatoria en España han sido, por lo general, muy amplias y difusas, con cierta vaguedad y poca delimitación (García Nieto y Cano, 2008). Se han analizado de una forma más pormenorizada en la Comunidad Autónoma de Aragón (Cano, 2006) y en el presente artículo vamos a comenzar haciendo un breve recorrido por diversas investigaciones y estudios que analizan de alguna forma la relación existente entre el trabajo profesional de los orientadores y las percepciones que el resto de la comunidad educativa tiene sobre éstos.

En primer lugar; nos centramos en analizar la opinión que tiene el alumnado sobre el trabajo profesional de los orientadores en el ámbito extranjero.

Por un lado, Hutchinson y Bottorff (1986) señalan que en la década de los ochenta y noventa, a pesar de las mejoras realizadas con los profesionales de la orientación, es decir, una mayor exigencia en su formación, en supervisión y en perfeccionamiento apenas existen diferencias en la visión que los estudiantes tienen sobre estos en dichas décadas. Por otro, Wells y Ritter (1979) propusieron a quinientos cincuenta estudiantes que cumplimentaran un cuestionario para detectar qué porcentaje de ellos acudirían al orientador para solicitar información o resolver alguna cuestión determinada. Entre los resultados, obtuvieron los siguientes:

Tabla 1. Porcentaje de alumnado que acudiría a un orientador para solicitar ayuda.

\begin{tabular}{|l|c|}
\hline Consultas & $\begin{array}{c}\text { Porcentaje de } \\
\text { alumnado }\end{array}$ \\
\hline Cambiar de clase & 81 \\
\hline Dudas sobre la graduación & 80 \\
\hline Planificación en los estudios & 51 \\
\hline Conflicto con el profesorado & 51 \\
\hline Elección de Universidad & 27 \\
\hline Problemas económicos & 27 \\
\hline Elección vocacional & 27 \\
\hline Elección de especialidad universitaria & 27 \\
\hline Conflictos familiares & 12 \\
\hline Orientación vocacional & 8 \\
\hline Graves problemas & 7 \\
\hline Problemas relacionales & 6 \\
\hline Sexo & 4 \\
\hline Problemas personales & 4 \\
\hline Búsqueda de empleo & 4 \\
\hline
\end{tabular}

Fuente: Wells y Ritter (1979) en Sanz (1999). 
Esta misma muestra de estudiantes, realizaban el siguiente ordenamiento de las funciones del orientador: Ayudar en la planificación del programa educativo; clarificar y planificar los estudios universitarios; asesorar sobre problemas personales; ayudar en la selección de la Universidad y Formación Profesional; orientación vocacional; trabajar con alumnado con problemas de disciplina; ayudar en la inserción laboral; charlas a familias, profesorado y alumnado; interpretar la información obtenida en los test; asesorar sobre la asistencia a clases y ejercer supervisión en el campus.

Por su parte, casi una cuarta parte de dichos estudiantes pensaban que el orientador cumplía un papel importante en muchas situaciones, algo más del $40 \%$ en la mayoría; y una tercera parte pensaban que no era una persona útil en el Centro. Respecto a la accesibilidad y cercanía del orientador, una tercera parte del alumnado pensaba que este profesional debería estar más cercano a su realidad. La mitad del alumnado pensaba que se podían sentir libres para hablar con el orientador mientras que la otra mitad opinaba lo contrario.

Resaltamos la investigación de Hutchinson y Bottorff (1986), quienes consultaron a doscientos cincuenta estudiantes del primer curso de la Universidad acerca de sus percepciones de los orientadores en Secundaria. Casi un 90\% había precisado orientación vocacional, aunque afirmaban que tan sólo un 40\% la había recibido. Ocho de cada diez necesitaba información sobre estudios universitarios, pero sólo la mitad la habían recibido. Un 60\% decía que había necesitado en algún momento orientación personal, mientras que tan sólo un $21 \%$ la había tenido. Por el contrario, algo más de la mitad había necesitado apoyo en su organización escolar, mientras que dicho apoyo lo habían tenido en mayor medida, un 78\%.

Otra investigación -Hutchinson y Reagan, 1989- encuestó a estudiantes de diez Centros de Indiana seleccionados al azar sobre cuarenta y ocho, con un instrumento con el que se incluían indistintamente funciones de un profesor, de un orientador o de un director, y se llegaba a la conclusión de que tampoco se tenía una visión clara de las funciones específicas de los orientadores.

Por su parte, Rowe (1989) recogió la opinión de una muestra de doscientos noventa y nueve estudiantes de la Bringham Young University de Utah concluyendo que la mayoría de orientadores no se había implicado suficientemente, según los estudiantes, en los planes de orientación profesional.

Sanz (1999) concreta respecto a la opinión del alumnado, en la dimensión del asesoramiento, que las alumnas dan más importancia que los alumnos a las funciones del orientador de ayudar a alguien que tiene problemas en sus relaciones familiares, en la adaptación de conducta escolar, en sus relaciones sociales o con problemas de aprendizaje en sus estudios.

También las alumnas dan más importancia que los alumnos, respecto a la dimensión II de la orientación con el grupo clase, a realizar en clase actividades que potencien los valores y las habilidades en las tomas de decisión; facilitar la comprensión y conocimiento de uno mismo; desarrollar destrezas de comunicación y relación entre el alumnado; la orientación vocacional; planificación de metas para su futuro profesional; utilizar los recursos humanos para conectar a los estudiantes con el mundo profesional y, finalmente, en proporcionar información sobre las alternativas al finalizar la ESO. Sin embargo, son los alumnos los que tienden a mani- 
festar más necesidad de recibir ayuda que las alumnas en cuanto a problemas de adaptación escolar.

Además del alumnado, encontramos referencias respecto al profesorado. Por ejemplo, Valine et al. (1982) escogieron a una muestra de cien y les pasaron un cuestionario de quince ítems analizando la percepción del profesorado hacia las funciones del orientador y comparándolo con otro estudio anterior en 1972. El 15\% del profesorado no tenía claro y presentaba serias dudas acerca de la pertinencia y necesidad de los orientadores; otro $35 \%$ no comprendía exactamente el rol de estos profesionales y cerca de un $60 \%$ veía regular la eficacia del trabajo del orientador. Son datos realmente preocupantes.

Por otro lado, Alaniz (1990) destacó en sus conclusiones que un 52\% del profesorado de los que respondieron al cuestionario, nunca o muy raramente consultaban con los orientadores sobre temas relacionados con la propia instrucción. Como aspecto más positivo, casi cuatro de cada diez orientadores comentaban y consultaban sobre problemática diversa del alumnado al menos con una frecuencia mensual. Otras conclusiones nos llevan a la idea de que el profesorado, en una gran parte, no conoce las funciones específicas de los orientadores ya que "aunque el $41 \%$ del profesorado afirmaba que los orientadores invertían mucho tiempo en asesorar -al alumnado y el $48 \%$ en tareas burocráticas, otro $40 \%$ de profesorado desconocía claramente dicho rol profesional" (Alaniz, 1990: 55).

Gibson (1990) recogió la opinión de ciento ochenta profesores que estaban ejerciendo al menos desde hace cinco años en Secundaria, de un total de diecinueve Centros. El asesoramiento individual era la función más importante del orientador. Entre los resultados, podemos destacar los siguientes:

Tabla 2. Percepción del profesorado sobre las funciones del orientador.

\begin{tabular}{|l|c|c|}
\hline \multirow{2}{*}{ Actividad } & \multicolumn{2}{|c|}{ Orden en la elección } \\
\cline { 2 - 3 } & $\mathbf{1 9 8 6}$ & $\mathbf{1 9 6 5}$ \\
\hline Información vocacional & 2 & 2 \\
\hline Control y registro de asistencia & 9,5 & 7 \\
\hline Disciplina & 8 & 6 \\
\hline Gestión e interpretación de tests & $>$ & 3 \\
\hline Tareas administrativas del programa tutorial & 9,5 & 9,5 \\
\hline Orientación individualizada & 1 & 1 \\
\hline Coordinación del programa de actividades de la escuela & 6 & 8 \\
\hline $\begin{array}{l}\text { Tutorías grupales, programas de asesoramiento y } \\
\text { orientación }\end{array}$ & 5 & 4 \\
\hline Gestión de plazas escolares & 4 & 9,5 \\
\hline $\begin{array}{l}\text { Gestión de puestos de trabajo, a media jornada y } \\
\text { jornada completa }\end{array}$ & 7 & 5 \\
\hline
\end{tabular}

Fuente. Gibson (1990: 523). 
Remarcamos el estudio de Sanz (1999) el cual recoge la opinión que distintos miembros de la comunidad educativa tienen respecto a las funciones de los orientadores. En relación con la opinión de los tutores, las profesoras respecto a los profesores piensan que los orientadores realizan con más frecuencia, dentro del asesoramiento, la función de ayudar a un estudiante que abandona los estudios en la búsqueda de otras alternativas y realizar estudios de investigación individuales o con orientadores de otros Centros. Respecto a la edad, Sanz recoge las conclusiones de que los tutores mayores de cuarenta años consideran que los orientadores realizan con mayor frecuencia ayudar, en grupos reducidos, a estudiantes con problemas de orientación vocacional; realizar estudios de seguimiento del proceso de transición de los alumnos de ESO a estudios posteriores y, finalmente, realizar estudios del proceso de transición del alumnado de Bachillerato a la Universidad.

Por su parte, los tutores perciben que los orientadores organizan pocas jornadas de acogida a los estudiantes de nuevo ingreso o facilitan asesoramiento a los programas comunitarios sobre civismo y ecología -educación vial o educación del medio ambiente. Las tutoras piensan que es importante que los orientadores, en lugar de ellas, ayuden a los estudiantes que presentan problemas de adaptación de conducta escolar o trabajen con el grupo-clase para facilitar en el alumnado la comprensión, el conocimiento y aceptación de sí mismo -autoconceptoy de los demás. Además, los profesores expertos piensan en mayor medida que los noveles que los orientadores ayuden a un estudiante que presenta problemas de drogas/alcohol.

Requiere de un análisis pormenorizado, las dimensiones planteadas en el instrumento diseñado por González (2007: 218-220) destacan en relación con "la disciplina, hábitos y técnicas de estudio, acción tutorial, atención a la diversidad, clima afectivo, evaluación/promoción, normativa, desarrollo personal, programas de orientación, orientación profesional, aspectos didácticos y participación de las familias".

Con una explicación media de la varianza del 79,21\%, González (2007: 221), de 24 elementos incluidos se obtienen los siguientes: asesoramiento al alumnado en la toma de decisiones, implicación de las familias, asesoramiento normativo sobre evaluación, participación en el diseño y desarrollo de las tutorías, orientación personal, elaboración de las normas de convivencia en el Centro, propuesta de nuevas medidas disciplinarias y diagnóstico de necesidades educativas especiales.

Entre las funciones más demandadas al orientador por parte del alumnado, siguiendo esta investigación son las que hacen referencia "a la toma de decisiones, desarroIlo de programas, acciones para implicar a las familias, información y asesoramiento sobre oferta educativa y profesional y recursos disponibles; que sea asesor del profesorado, participante activo en el diseño del plan de acción tutorial, específicamente en hábitos y técnicas de estudio; desarrollo de estilos de vida saludable, planes de convivencia y asesoramiento al equipo docente en dificultades de aprendizaje" (González, 2007:225). Continúa señalando que el alumnado opina que las funciones más relevantes del orientador son "el desarrollo de programas de educación para la salud, ayuda al alumnado en su toma de decisiones, fomento de un clima adecuado de cen- 
tro, asesoramiento sobre legislación, coordinar la atención a la diversidad, acciones para favorecer la participación de las familias en el proceso educativo, asesoramiento sobre investigación e innovación educativa y metodologías didácticas, diseño del plan de acción tutorial, diagnóstico de las dificultades de aprendizaje y asesoramiento sobre evaluación". Como observamos, el espectro de funciones es muy amplio y disperso.

Respecto a las funciones más demandadas de las familias hacia el orientador, concluye González (2007:226) que son "la orientación profesional, favorecer el clima afectivo del centro, coordinar el diseño y desarrollo de las tutorías, dinamitar programas de educación para la salud, intervención en la implementación de las normas de convivencia, fomente su implicación en la educación, asesoramiento legal y cómo favorecer hábitos y técnicas de estudio.

Las funciones más demandadas del orientador por parte de los equipos directivos son "el asesoramiento para favorecer la convivencia en el Centro, medidas de atención a la diversidad y principios didácticos" (González, 2007:227). En este sentido, observamos cómo determinadas funciones se han ido repitiendo por parte de los diferentes miembros de la comunidad educativa y van confluyendo, aunque no están contrastados los datos con la importancia real que atribuyen los propios profesionales.

Resulta importante, tal y corno hemos ido observando, detectar algunas de las tendencias prioritarias en los escasos estudios que se han abordado acerca de esta temática en cuestión, con la interrelación entre la comunidad educativa y el trabajo profesional de los orientadores.

\section{MÉTODO}

La finalidad que se propone este trabajo es realizar un análisis de la importancia de las funciones según lo que piensan los orientadores en Aragón, centrándose en la etapa de la Educación Secundaria Obligatoria respecto a las percepciones de la comunidad educativa.

Las variables en las que nos hemos centrado para realizar dicho análisis son la coordinación, el diagnóstico, la docencia, la evaluación/innovación e investigación, la formación, la información y la intervención psicopedagógica tal y como vemos a continuación en la segunda pregunta del Cuestionario para el Análisis de las Funciones de los Orientadores (CAFO) que se ha diseñado ad hoc, tal y como puede ampliarse en Cano (2006) y García Nieto y Cano (2008). Es de destacar que obtuvimos un resultado muy positivo mediante el Alfa de Cronbach $(0,97)$ y fue revisado por un grupo de expertos y un grupo piloto, con los que se obtuvo finalmente una media superior a cuatro sobre cinco en la claridad y pertinencia del instrumento. Hemos podido analizar los diferentes porcentajes en las respuestas, tras haberlas agrupado (nada-poco, bastante-mucho) así como las diferencias significativas.

Hemos obtenido respuesta de más del $80 \%$ de los orientadores de Aragón, que todos ellos ascienden a 175, si bien, seis de ellos desempeñan sus funciones en dos centros al mismo tiempo. 
PERCEPCIÓN DEL ORIENTADOR ACERCA DE LA IMPORTANCIA QUE ATRIBUYE LA COMUNIDAD EDUCATIVA...

\section{Tabla 3.}

Contesta, según la escala 1. Ninguna, 2. Poca, 3. Regular, 4. Bastante, 5. Mucha, la importancia que en tu opinión atribuye cada colectivo a cada una de tus funciones.

\begin{tabular}{|l|l|l|l|l|l|l|}
\hline \multirow{2}{*}{ Funciones } & \multicolumn{5}{|c|}{ Importancia que atribuye cada colectivo } \\
\cline { 2 - 7 } & Familias & Alumnado & Profesorado & Tutores & Dirección & Administración \\
\hline $\begin{array}{l}\text { 1. Coordinación de la } \\
\text { orientación }\end{array}$ & & & & & & \\
\hline 2. Diagnóstico & & & & & & \\
\hline 3. Docencia & & & & & & \\
\hline $\begin{array}{l}\text { 4. Evaluación, innovación e } \\
\text { investigación }\end{array}$ & & & & & & \\
\hline $\begin{array}{l}\text { 5. Formación a la comunidad } \\
\text { educativa }\end{array}$ & & & & & & \\
\hline $\begin{array}{l}\text { 6. Información-asesoramiento a } \\
\text { la comunidad educativa }\end{array}$ & & & & & & \\
\hline $\begin{array}{c}\text { 7. Intervención en el plan de } \\
\text { acción tutorial }\end{array}$ & & & & & & \\
\hline $\begin{array}{l}\text { 8. Intervención en el plan de } \\
\text { atención a la diversidad }\end{array}$ & & & & & & \\
\hline $\begin{array}{c}\text { 9. Orientación académica y } \\
\text { profesional }\end{array}$ & & & & & & \\
\hline
\end{tabular}

\section{Resultados}

A continuación, vamos a describir las percepciones que tienen los orientadores acerca de la importancia que creen que atribuyen las familias, el alumnado, el profesorado, la Dirección y la Administración acerca de sus funciones.

Comenzamos señalando la percepción que tienen los orientadores acerca de la importancia que atribuyen las familias a sus funciones.

Tabla 4. Valoración del orientador sobre la importancia que atribuyen las familias a sus funciones.

\begin{tabular}{|l|c|c|c|c|}
\hline Funciones & $\begin{array}{c}\text { Ninguna/ } \\
\text { Poca }\end{array}$ & Regular & $\begin{array}{c}\text { Bastante/ } \\
\text { Mucha }\end{array}$ & N \\
\hline Coordinación de la orientación & 43,94 & 24,24 & 31,82 & 132 \\
\hline Diagnóstico & 7,46 & 22,39 & 70,15 & 134 \\
\hline Docencia & 28,21 & 30,77 & 41,03 & 117 \\
\hline Evaluación, innovación e investigación & 64,29 & 20,63 & 15,08 & 126 \\
\hline Formación a la comunidad educativa & 33,59 & 32,81 & 33,59 & 128 \\
\hline Información/asesoramiento a la comunidad educativa & 11,45 & 22,90 & 65,65 & 131 \\
\hline Intervención en el Plan de Acción Tutorial & 23,66 & 40,46 & 35,88 & 131 \\
\hline Intervención en el Plan de Atención a la Diversidad & 18,46 & 29,23 & 52,31 & 130 \\
\hline Orientación académica y profesional & 4,44 & 8,89 & 86,67 & 135 \\
\hline
\end{tabular}


Los mayores porcentajes de orientadores que opinan que las familias dan bastante/mucha importancia a sus funciones son los siguientes, tal y como se recoge en la Tabla $n^{\circ}$ 4: 86,67\% a la orientación académica y profesional; $70,15 \%$ al diagnóstico y $65,65 \%$ a la información. Posiblemente, con estos datos se puede ver cómo los orientadores perciben que las familias necesitan de la escuela -tutores, profesorado y Departamento de Orientación- para orientar a sus hijos en los distintos itinerarios académicos y orientarles vocacionalmente. En segundo lugar, el diagnóstico puede ser percibido por la familia como una ayuda adicional para detectar las dificultades de aprendizaje o de comportamiento de sus hijos. Los orientadores, perciben, en tercer lugar, que la transmisión de información es importante en la relación con las familias.

Por su parte, los orientadores creen que las familias no dan ninguna/poca importancia a su función de evaluación/innovación/investigación $(64,29 \%)$, posiblemente porque éstas quieren ver resultados prácticos y concretos que haga que sus hijos aprendan más y mejor en un corto período de tiempo. Por otro lado, una tercera parte de los orientadores piensa que las familias dan ninguna/poca importancia a la formación de la comunidad educativa, porcentaje alto que puede reflejar cómo la función formativa no ha calado suficientemente en los centros. Si los propios orientadores perciben que las familias son las que valoran poco la formación; las que no la aprecian ni la aprovechan; las que cuando se les propone alguna reunión no van a acudir, en consecuencia serán los orientadores los que programen pocas actuaciones relacionadas con la formación de las familias.

Otro de los retos educativos fundamentales en los albores del siglo XXI, es precisamente el de la atención a la diversidad. Cabe constatar con relación a este aspecto que casi un $20 \%$ piensa que las familias dan ninguna/poca importancia a esta función tan actual y urgente en nuestro Sistema Educativo, para atender a cada estudiante de forma diferente, ya que distintas son sus motivaciones, expectativas y aptitudes.

Cerca del 23,66\% de los orientadores cree que las familias dan ninguna/poca importancia a la intervención en el Plan de Acción Tutorial. En este sentido, los orientadores no ven valorado su apoyo y colaboración en desarrollar e implantar nuevas propuestas en la tutoría, aspecto que posteriormente a nivel universitario está cobrando mayor relevancia (García Nieto et al., 2005). Cabe preguntarse hasta qué punto las familias dan mayor o menor importancia realmente a la tutoría de sus hijos. O, por otro lado, si valoran en mayor medida que sus hijos adquieran muchos conocimientos, altas calificaciones en las diversas áreas y releguen para un segundo plano, la adquisición de otras actitudes que se cultivan con la tutoría. También podríamos analizar si esta percepción negativa de los orientadores acerca de lo que piensan las familias sobre la tutoría; coincide al estar hablando de una tutoría ya en sí misma desprestigiada, poco valorada e incentivada, dedicada en muchas ocasiones a actividades alternativas de estudio, a tiempo libre sin clase propiamente dicha y marginada en el horario, concentrándose en los viernes como último día de la semana, o a última hora de la mañana o, sencillamente, por las tardes en aquellos centros que tienen este tipo de horario. 
PERCEPCIÓN DEL ORIENTADOR ACERCA DE LA IMPORTANCIA QUE ATRIBUYE LA COMUNIDAD EDUCATIVA...

Tabla 5. Valoración del orientador sobre la importancia que atribuye el alumnado a sus funciones.

\begin{tabular}{|l|c|c|c|c|}
\hline Funciones & $\begin{array}{c}\text { Ninguna/ } \\
\text { Poca }\end{array}$ & Regular & $\begin{array}{c}\text { Bastante/ } \\
\text { Mucha }\end{array}$ & N \\
\hline Coordinación de la orientación & 44,27 & 23,66 & 32,06 & 131 \\
\hline Diagnóstico & 20,30 & 38,35 & 41,35 & 133 \\
\hline Docencia & $\mathbf{1 4 , 6 6}$ & 34,48 & 50,86 & 116 \\
\hline Evaluación, innovación e investigación & $\mathbf{6 1 , 7 2}$ & 23,44 & 14,84 & 128 \\
\hline Formación a la comunidad educativa & $\mathbf{5 2 , 3 4}$ & 32,81 & 14,84 & 128 \\
\hline Información/asesoramiento a la comunidad educativa & 25,38 & 28,46 & 46,15 & 130 \\
\hline Intervención en el Plan de Acción Tutorial & 23,48 & 34,09 & 42,42 & 132 \\
\hline Intervención en el Plan de Atención a la Diversidad & 20,61 & 34,35 & 45,04 & 131 \\
\hline Orientación académica y profesional & 3,70 & 7,41 & $\mathbf{8 8 , 8 9}$ & 135 \\
\hline
\end{tabular}

Otro aspecto relevante que analizamos a continuación es sobre la percepción de los orientadores acerca de la importancia que puedan atribuir el alumnado a sus funciones. En este sentido, piensa que el alumnado da bastante/mucha importancia a la orientación académica y profesional. Éstos necesitan recabar información de las distintas alternativas a lo largo de sus estudios, en la elección de asignaturas optativas, itinerarios, tipo de estudios futuros, relacionados con los Programas de Garantía Social, los Ciclos Formativos de Grado Medio y Superior, con la formación universitaria o para incorporarse al mundo laboral. A más distancia, observamos que el $50 \%$ de los orientadores piensa que el alumnado da bastante/mucha importancia a que den clase. Posiblemente, la mitad de los orientadores, poniéndose en el lugar de los estudiantes, perciben que para ellos dar clase es importante ya que de esta manera, pueden estar más cercanos a los problemas concretos del alumnado, pueden ser mejor conocidos por éstos y no estar más separados o aislados en el despacho, o etiquetados más bien como el profesional encargado de atender a aquéllos alumnos con serias dificultades y con graves problemas. Puede ser una manera de normalizar dicho rol.

Por el contrario, también podemos analizar cómo sólo un 15\% dan ninguna/poca importancia a su función docente. Dicha opinión puede deberse a que ya tienen demasiadas funciones y trabajo burocrático encomendado, como para dedicar tanto tiempo como el que les exige la Administración en tareas docentes. En opinión de un orientador, se constaba lo siguiente: "porque se nos quiere considerar un profesor más, con las funciones docentes como prioritarias". Es posible también, que estos datos reflejen que una parte del alumnado pueda pensar que si el orientador imparte docencia, tiene "demasiado que ver" con el rol evaluador del profesor. En este sentido, un orientador que no califica, que está más distanciado de la evaluación y que mantiene su independencia, sin estar contaminado por otras variables más relacionadas con el proceso de enseñanza-aprendizaje, estos alumnos podrían sentirse más libres y motivados para consultar, ya que el orientador posiblemente no tenga una imagen preconcebida de ellos. Esto mismo puede tener algún inconveniente más, en 
el sentido de que el orientador carece de ese contacto casi diario con el alumnado, que le permita conocer más directamente sus intereses, su forma de ser, sus inquietudes o su personalidad, con lo que la información que pueda impartir a nivel de orientación no se adapte totalmente a la casuística de dicho estudiante. En muchas ocasiones, el alumno se comporta diferente en el contexto de su clase que en una entrevista personal en el Departamento de Orientación.

La información no obtiene datos muy altos desde la perspectiva del orientador hacia el alumnado. Nos Ilama la atención cómo uno de cada cuatro orientadores da ninguna/poca importancia a dicha función. Posiblemente el alumnado no ve suficientemente claro que el Departamento de Orientación sea un lugar de recogida de información. Esto puede tener que ver, en ocasiones, con una escasa motivación del alumnado en cuanto a inquietudes. Pero no nos queremos quedar en un análisis simplista o reduccionista de la realidad. Diversos Departamentos de Orientación hacen fluir la información a través de los tutores de una manera ágil y eficaz. Otros, ciertamente, no tanto. Y de entre estos tutores, un grupo facilitará al alumnado dicha información de manera motivante y atractiva, mientras que otros se limiten a dejar dicha información en la mesa del profesor o en el corcho del pasillo o de clase, en el que apenas se le da importancia. Nos parece importante señalar en estas líneas qué está pasando realmente con la información que recoge el Departamento de Orientación y qué pueda interesar más al alumnado.

Por otro lado, la evaluación, innovación e investigación recoge el 61\% de las respuestas en las que el orientador percibe que el alumnado da ninguna/poca importancia. Éstos perciben muy distante esta función. En segundo lugar, algo más de la mitad de los orientadores piensa que el alumnado da ninguna/poca importancia a su función de formación, dato que resulta muy valorado.

En el siguiente apartado, recogemos algunos datos referentes a las percepciones que tienen los orientadores acerca de la importancia que atribuye el profesorado a sus funciones.

Tabla 6. Valoración del orientador sobre la importancia que atribuye el profesorado a sus funciones.

\begin{tabular}{|l|c|c|c|c|}
\hline Funciones & $\begin{array}{c}\text { Ninguna/ } \\
\text { Poca }\end{array}$ & Regular & $\begin{array}{c}\text { Bastante/ } \\
\text { Mucha }\end{array}$ & N \\
\hline Coordinación de la orientación & 15,79 & 32,33 & 51,88 & 133 \\
\hline Diagnóstico & 4,51 & 17,29 & 78,20 & 133 \\
\hline Docencia & 19,83 & 39,66 & 40,52 & 116 \\
\hline Evaluación, innovación e investigación & 33,08 & 39,23 & 27,69 & 130 \\
\hline Formación a la comunidad educativa & 25,78 & 42,19 & 32,03 & 128 \\
\hline Información/asesoramiento a la comunidad educativa & 13,74 & 24,43 & 61,83 & 131 \\
\hline Intervención en el Plan de Acción Tutorial & 9,85 & 30,30 & 59,85 & 132 \\
\hline Intervención en el Plan de Atención a la Diversidad & 3,85 & 19,23 & 76,92 & 130 \\
\hline Orientación académica y profesional & 2,99 & 17,16 & 79,85 & 134 \\
\hline
\end{tabular}


Alrededor del $80 \%$ de los orientadores coinciden en que el profesorado da bastante/mucha importancia a la orientación académica y profesional, al diagnóstico y a la intervención en el Plan de Atención a la Diversidad. Posiblemente, podríamos decir que son tres ámbitos específicos del orientador y que el profesorado en general carece de la información suficiente para desarrollar convenientemente estos aspectos. En este sentido, es precisa la estrecha colaboración entre el orientador y el profesorado para facilitar o posibilitar una mejora cualitativa en el proceso de enseñanza-aprendizaje. Una educación de calidad no puede dejar de lado, de forma marginada ni delegada en alguien en exclusiva la importante labor de atender a los diferentes alumnos que están en las aulas. La cultura de colaboración, el trabajo en equipo, la coordinación, la información y la formación se vislumbran como requisitos necesarios para llevar a cabo convenientemente esta atención. La evaluación, innovación e investigación, como hemos señalado en anteriores epígrafes, recibe también la valoración menor de acuerdo en cuanto a la importancia que tiene. Se sitúa, junto a la formación, alrededor de un 30\% de orientadores que piensan que el profesorado le da bastante/mucha importancia y también una tercera parte que le da ninguna/poca. Esta última, la formación, ofrece unos datos cuanto menos preocupantes.

Podemos añadir también cómo uno de cada cuatro orientadores percibe que el profesorado da ninguna/poca importancia a la formación a la comunidad educativa. En este sentido, creemos que es muy importante que se mejore la colaboración y el trabajo en equipo entre el orientador y el profesorado, puesto que ambos tienen un objetivo común que es brindar una educación de calidad al alumnado. Quizás, de estos datos, puedan deducirse algunas reticencias a la formación, a la actualización o al reciclaje por parte de algunos, hecho que dificulta que dicha función cuaje en su totalidad.

Los orientadores, por su parte, consideran la opinión de los tutores acerca de sus funciones de la siguiente manera, analizando los datos obtenidos en la Comunidad Aragonesa.

Tabla 7. Valoración del orientador sobre la importancia que atribuyen los tutores a sus funciones.

\begin{tabular}{|l|c|c|c|c|}
\hline Funciones & $\begin{array}{c}\text { Ninguna/ } \\
\text { Poca }\end{array}$ & Regular & $\begin{array}{c}\text { Bastante/ } \\
\text { Mucha }\end{array}$ & N \\
\hline Coordinación de la orientación & 6,77 & 15,79 & 77,44 & 133 \\
\hline Diagnóstico & 0,75 & 13,43 & 85,82 & 134 \\
\hline Docencia & 15,52 & 39,66 & 44,83 & 116 \\
\hline Evaluación, innovación e investigación & 27,91 & 37,98 & 34,11 & 129 \\
\hline Formación a la comunidad educativa & 19,38 & 37,21 & 43,41 & 129 \\
\hline Información/asesoramiento a la comunidad educativa & 9,92 & 16,79 & 73,28 & 131 \\
\hline Intervención en el Plan de Acción Tutorial & 1,52 & 14,39 & 84,09 & 132 \\
\hline Intervención en el Plan de Atención a la Diversidad & 3,79 & 10,61 & 85,61 & 132 \\
\hline Orientación académica y profesional & 1,49 & 2,24 & 96,27 & 134 \\
\hline
\end{tabular}


Un 96,21\% de los orientadores piensa que los tutores dan bastante/mucha importancia a la orientación académica y profesional. Y, alrededor del 85\% coincide también en dar esta misma importancia al diagnóstico y a la intervención en el Plan de Atención a la Diversidad. Son, en este sentido, las mismas tres funciones que tienen los primeros puestos con relación a lo que el profesorado piensa. En el caso de los tutores dicho porcentaje aumenta en unos diez puntos aproximadamente, Ilegando a puntuaciones muy altas. También este colectivo añade, a diferencia del profesorado, la importancia de la intervención' en el Plan de Acción Tutorial. Los tutores alcanzan un $84 \%$ y el profesorado un $60 \%$, lo cual puede deberse, evidentemente, a que son los tutores los encargados y responsables más directos de desarrollar específicamente la tutoría.

Por su parte, los orientadores, al igual que en el caso del profesorado, piensan que los tutores dan poco/nada de importancia a la evaluación, innovación e investigación $(27,91)$ y a la formación $(19,38)$, si bien bajan seis puntos con relación al profesorado. Cerca del $40 \%$ piensan que la importancia que dan dichas funciones es regular, ni se decantan en sentido positivo ni negativo.

Analizamos a continuación la percepción de los orientadores sobre la visión de la Dirección.

Tabla 8. Valoración del orientador sobre la importancia que atribuye la dirección a sus funciones.

\begin{tabular}{|l|c|c|c|c|}
\hline Funciones & $\begin{array}{c}\text { Ninguna/ } \\
\text { Poca }\end{array}$ & Regular & $\begin{array}{c}\text { Bastante/ } \\
\text { Mucha }\end{array}$ & N \\
\hline Coordinación de la orientación & 3,82 & 17,56 & 78,63 & 131 \\
\hline Diagnóstico & 8,40 & 11,45 & 80,15 & 131 \\
\hline Docencia & 8,77 & 32,46 & 58,77 & 114 \\
\hline Evaluación, innovación e investigación & 27,34 & 31,25 & 41,41 & 128 \\
\hline Formación a la comunidad educativa & 19,69 & 24,41 & 55,91 & 127 \\
\hline Información/asesoramiento a la comunidad educativa & 9,23 & 14,62 & 76,15 & 130 \\
\hline Intervención en el Plan de Acción Tutorial & 3,08 & 16,15 & 80,77 & 130 \\
\hline Intervención en el Plan de Atención a la Diversidad & 3,85 & 10,77 & 85,38 & 130 \\
\hline Orientación académica y profesional & 2,27 & 8,33 & 89,39 & 132 \\
\hline
\end{tabular}

Por otro lado, el 90\% de los orientadores coincide en señalar que la Dirección del Centro da bastante/mucha importancia a la orientación académica y profesional, seguido de un $86 \%$ que da mucho valor a la intervención en el Plan de Atención a la Diversidad. Posteriormente, alrededor de un 80\%, aproximadamente, de orientadores considera que la Dirección da bastante/mucha importancia a la intervención en el Plan de Acción Tutorial, la coordinación, el diagnóstico y la información.

Los orientadores tienen prácticamente igual percepción en cuanto a la poca/ninguna importancia que da la Dirección a las funciones menos valoradas. Dichas fun- 
ciones siguen siendo la evaluación, innovación e investigación $(27,34)$ y la formación con cerca del $20 \%$.

Finalmente, los orientadores perciben que la Administración valora mucho menos su trabajo, dándole escasa importancia a sus funciones comparando con la comunidad educativa propiamente dicha, formada por las familias, el alumnado, el profesorado, los tutores y los equipos directivos. Cabe señalar que la relación entre el orientador y la propia Administración tiene una capital importancia. De los vínculos y de la colaboración entre éstos radica, en buena parte, el mayor o menor grado de satisfacción laboral.

Tabla 9. Valoración del orientador sobre la importancia que atribuye la Administración a sus funciones.

\begin{tabular}{|l|c|c|c|c|}
\hline Funciones & $\begin{array}{c}\text { Ninguna/ } \\
\text { Poca }\end{array}$ & Regular & $\begin{array}{c}\text { Bastante/ } \\
\text { Mucha }\end{array}$ & N \\
\hline Coordinación de la orientación & 32,52 & 32,52 & 34,96 & 123 \\
\hline Diagnóstico & 24,30 & 40,19 & 35,51 & 107 \\
\hline Docencia & 20,69 & 23,28 & 56,03 & 116 \\
\hline Evaluación, innovación e investigación & 44,44 & 32,48 & 23,08 & 117 \\
\hline Formación a la comunidad educativa & 40,17 & 34,19 & 25,64 & 117 \\
\hline Información/asesoramiento a la comunidad educativa & 27,97 & 35,59 & 36,44 & 118 \\
\hline Intervención en el plan de acción tutorial & 21,01 & 34,45 & 44,54 & 119 \\
\hline Intervención en el plan de atención a la diversidad & 16,39 & 26,23 & 57,38 & 122 \\
\hline Orientación académica y profesional & 15,83 & 26,67 & 57,50 & 120 \\
\hline
\end{tabular}

El primer análisis que hacemos, en este sentido, son los porcentajes menores que, en general, se registran en el apartado de bastante/mucha importancia atribuido a cada una de las diferentes funciones.

Además, podemos señalar que la muestra de orientadores que responden a esta pregunta referida a la Administración es menor. Se sitúa en una media de ciento quince orientadores. Una de las posibles causas que podemos comentar es un cierto distanciamiento que pueden sentir los orientadores respecto a la Administración, unido a una cierta dejadez de ésta y a una sobrecarga en las funciones y en el trabajo, que hace que estos profesionales no estén muy satisfechos en su trabajo. Por otro lado, algunos Centros Concertados también han señalado que apenas mantienen relación con la Administración, sintiéndose desatendidos. Haciendo un análisis de los datos, podemos comenzar diciendo en este apartado que no llega al $60 \%$ el número de orientadores que percibe que la Administración da bastante/mucha importancia a la orientación académica y profesional, a la intervención en el Plan de Atención a la Diversidad y al diagnóstico, siendo éstas las funciones mejor consideradas. Tan sólo uno de cada cuatro orientadores percibe que la Administración considera como bastante o muy importante desarrollar su función de formar a la 
comunidad educativa -cabe resaltar cómo un 40\% piensa que la Administración da ninguna o poca importancia- y un $23 \%$ de los orientadores que señalan que la evaluación, innovación e investigación es bastante/muy importante, frente a casi un $45 \%$ que considera que se le da ninguna o poca importancia.

La opinión de los orientadores acerca de cómo la Administración valora su función de coordinación, se reparte proporcionalmente en tres terceras partes, es decir: Un primer grupo piensa que la Administración no valora o valora poco la coordinación; otra parte, prácticamente igual, señala que lo valora regular; y la tercera y última señala que se valora bastante o mucho. Los orientadores, pues, tienen una visión muy desigual con relación a la valoración que hace la Administración sobre la función de coordinación. Puede parecer que no hay mecanismos claros con relación a ciertas dimensiones de la coordinación de la orientación, aspecto que puede incidir posteriormente en el sentimiento de soledad o aislamiento del orientador.

Presentamos a continuación la tabla en la que aparecen las diferencias significativas en la importancia que da la comunidad educativa a las funciones del orientador según la edad.

Tabla 10. Diferencias significativas en la importancia que da la comunidad educativa a las funciones del orientador según edad.

\begin{tabular}{|l|c|c|c|c|c|}
\hline \multirow{2}{*}{ Importancia de las funciones } & \multicolumn{5}{|c|}{ Edad } \\
\cline { 2 - 6 } & $\begin{array}{c}\text { Menos de } \\
\mathbf{3 5} \text { años }\end{array}$ & $\begin{array}{c}\mathbf{3 5 - 5 0} \\
\text { años }\end{array}$ & $\begin{array}{c}\text { Más de } \\
\mathbf{5 0} \text { años }\end{array}$ & $\mathbf{F}$ & Sig. \\
\hline Docencia según el profesorado & 3,07 & 3,45 & 2,75 & $\mathbf{3 , 8 6 1}$ &, 025 \\
\hline Formación según los tutores & 3,61 & 3,28 & 2,65 & $\mathbf{5 , 8 9 1}$ &, 004 \\
\hline Formación según la Dirección & 3,94 & 3,44 & 2,88 & $\mathbf{4 , 8 9 9}$ &, 009 \\
\hline Información-asesoramiento según las familias & 4,16 & 3,60 & 3,29 & $\mathbf{5 , 9 7 7}$ &, 004 \\
\hline Información-asesoramiento según Dirección & 4,23 & 4,04 & 3,50 & $\mathbf{3 , 1 9 8}$ &, 045 \\
\hline
\end{tabular}

Respecto a las diferencias significativas existentes entre los miembros componentes de la muestra, señalamos en primer lugar, que hay una tendencia a que los orientadores entre 35-50 años piensan que el profesorado da más importancia a la docencia que los orientadores mayores de 50 años. Uno de los aspectos controvertidos y que está sujeto a variadas opiniones es la carga docente que debe o no debe tener el orientador. En este sentido, existe la opinión de que el orientador debería impartir algo de docencia, en áreas más propias y afines a su especialidad, pero que no le sobrecargue excesivamente en el horario. De este modo, podría tener una relación más fluida y cercana con el profesorado. Dicho de otra forma, con mayor legitimidad en el asesoramiento a este colectivo. 
PERCEPCIÓN DEL ORIENTADOR ACERCA DE LA IMPORTANCIA QUE ATRIBUYE LA COMUNIDAD EDUCATIVA...

Tabla 11. Diferencias significativas en la importancia que da la comunidad educativa a sus funciones con relación a la edad.

\begin{tabular}{|c|c|c|c|c|c|c|c|c|}
\hline \multirow{2}{*}{ Variable dependiente } & \multicolumn{3}{|c|}{ Comparaciones múltiples } & \multirow{2}{*}{$\begin{array}{c}\text { Diferencia } \\
\text { de } \\
\text { medias } \\
(\mathrm{I}-\mathrm{J})\end{array}$} & \multirow{2}{*}{$\begin{array}{l}\text { Error } \\
\text { típico }\end{array}$} & \multirow[b]{2}{*}{ Sig. } & \multicolumn{2}{|c|}{$\begin{array}{c}\text { Intervalo de } \\
\text { confianza al } 95 \%\end{array}$} \\
\hline & & (I) Edad & (J) Edad & & & & $\begin{array}{l}\text { Límite } \\
\text { inferior }\end{array}$ & $\begin{array}{l}\text { Límite } \\
\text { superior }\end{array}$ \\
\hline \multirow{6}{*}{$\begin{array}{l}\text { Importancia de la docencia } \\
\text { según profesorado }\end{array}$} & \multirow[t]{6}{*}{ Scheffé } & $<$ de 35 años & 35-50 años & $-0,375$ & 0,223 & 0,248 & $-0,930$ & 0,180 \\
\hline & & & $>$ de 50 años & 0,324 & 0,293 & 0,546 & $-0,406$ & 1,055 \\
\hline & & 35-50 años & $<$ de 35 años & 0,375 & 0,223 & 0,248 & $-0,180$ & 0,930 \\
\hline & & & $>$ de 50 años & 0,699 & 0,268 & 0,038 & 0,032 & 1,366 \\
\hline & & $>$ de 50 años & $<$ de 35 años & $-0,324$ & 0,293 & 0,546 & $-1,055$ & 0,406 \\
\hline & & & 35-50 años & $-0,699$ & 0,268 & 0,038 & $-1,366$ & $-0,032$ \\
\hline \multirow{6}{*}{$\begin{array}{l}\text { Importancia de la formación } \\
\text { según tutores }\end{array}$} & \multirow[t]{6}{*}{ Scheffé } & $<$ de 35 años & 35-50 años & 0,335 & 0,210 & 0,285 & $-0,187$ & 0,857 \\
\hline & & & $>$ de 50 años & 0,966 & 0,281 & 0,004 & 0,266 & 1,665 \\
\hline & & 35-50 años & $<$ de 35 años & $-0,335$ & 0,210 & 0,285 & $-0,857$ & 0,187 \\
\hline & & & $>$ de 50 años & 0,631 & 0,259 & 0,057 & $-0,014$ & 1,275 \\
\hline & & $>$ de 50 años & $<$ de 35 años & $-0,966$ & 0,281 & 0,004 & $-1,665$ & $-0,266$ \\
\hline & & & 35-50 años & $-0,631$ & 0,259 & 0,057 & $-1,275$ & 0,014 \\
\hline \multirow{6}{*}{$\begin{array}{l}\text { Importancia de la formación } \\
\text { según Dirección }\end{array}$} & \multirow[t]{6}{*}{ Scheffé } & $<$ de 35 años & 35-50 años & 0,491 & 0,252 & 0,156 & $-0,136$ & 1,118 \\
\hline & & & $>$ de 50 años & 1,060 & 0,345 & 0,011 & 0,203 & 1,918 \\
\hline & & 35-50 años & $<$ de 35 años & $-0,491$ & 0,252 & 0,156 & $-1,118$ & 0,136 \\
\hline & & & $>$ de 50 años & 0,569 & 0,319 & 0,208 & $-0,223$ & 1,362 \\
\hline & & $>$ de 50 años & $<$ de 35 años & $-1,060$ & 0,345 & 0,011 & $-1,918$ & $-0,203$ \\
\hline & & & 35-50 años & $-0,569$ & 0,319 & 0,208 & $-1,362$ & 0,223 \\
\hline \multirow{6}{*}{$\begin{array}{l}\text { Importancia de la } \\
\text { información/asesoramiento } \\
\text { según familia }\end{array}$} & \multirow[t]{6}{*}{ Scheffé } & $<$ de 35 años & 35-50 años & 0,561 & 0,205 & 0,026 & 0,053 & 1,069 \\
\hline & & & $>$ de 50 años & 0,867 & 0,275 & 0,009 & 0,184 & 1,550 \\
\hline & & 35-50 años & $<$ de 35 años & $-0,561$ & 0,205 & 0,026 & $-1,069$ & $-0,053$ \\
\hline & & & $>$ de 50 años & 0,306 & 0,253 & 0,483 & $-0,322$ & 0,934 \\
\hline & & $>$ de 50 años & $<$ de 35 años & $-0,867$ & 0,275 & 0,009 & $-1,550$ & $-0,184$ \\
\hline & & & 35-50 años & $-0,306$ & 0,253 & 0,483 & $-0,934$ & 0,322 \\
\hline \multirow{6}{*}{$\begin{array}{l}\text { Importancia de la } \\
\text { información/asesoramiento } \\
\text { según Dirección }\end{array}$} & \multirow[t]{6}{*}{ Scheffé } & $<$ de 35 años & 35-50 años & 0,189 & 0,211 & 0,669 & $-0,335$ & 0,714 \\
\hline & & & $>$ de 50 años & 0,726 & 0,289 & 0,047 & 0,007 & 1,444 \\
\hline & & 35-50 años & $<$ de 35 años & $-0,189$ & 0,211 & 0,669 & $-0,714$ & 0,335 \\
\hline & & & > de 50 años & 0,536 & 0,267 & 0,138 & $-0,127$ & 1,199 \\
\hline & & $>$ de 50 años & $<$ de 35 años & $-0,726$ & 0,289 & 0,047 & $-1,444$ & $-0,007$ \\
\hline & & & 35-50 años & $-0,536$ & 0,267 & 0,138 & $-1,199$ & 0,127 \\
\hline
\end{tabular}

Por el contrario, existe también la opinión de que la impartición de docencia lo que hace es cargar al orientador con otras tareas que son muy diferentes de las específicas de su especialidad en psicología y/o pedagogía. También puede influir en los datos obtenidos ciertas opiniones de algunos profesionales que piensan que impartir docencia es más laborioso que atender de forma más individualizada a determinado alumnado y sus familias. De este modo, aquellos orientadores mayores de 50 
años podrían dejar entrever cierto cansancio ante la docencia de forma más directa con el grupo de alumnos, fruto de cierto cansancio o rutina, y de las manifestaciones problemáticas que la adolescencia puede llevar consigo.

En segundo lugar, los orientadores menores de treinta y cinco años, piensan significativamente, que los tutores dan más importancia a la función de la formación que los orientadores mayores. Puede existir, en esta línea, una creciente cultura colaborativa entre los orientadores más jóvenes y los tutores, ya que de lo contrario, un orientador aislado, evidentemente, tiene una eficacia mucho más relativa en sus actuaciones en el centro. Por otro lado, una vez más, aquellos de mayor edad, posiblemente, han constatado, en mayor o menor medida, que el trabajo en común con el equipo de tutores no es tan sencillo o tan satisfactorio como a priori se esperaba.

Datos muy semejantes al apartado anterior, son los que recogemos respecto a la opinión que tienen los orientadores referida a la formación que deberían dar al equipo directivo.

Observamos que existen diferencias significativas en la mayor importancia que los orientadores más jóvenes piensan que otorgan las familias a la informaciónasesoramiento, respecto a los orientadores mayores de cincuenta años, y con una tendencia también mayor respecto a los que se encuentran en la franja de edad entre los 35-50 años. Una vez más, vemos que los orientadores más jóvenes piensan que es importante estrechar los lazos de comunicación entre la institución familiar y el Centro.

En segundo lugar, aunque con datos que habría que considerar con más cautela, vemos que existe una ligera tendencia por parte de los orientadores más jóvenes, respecto a los más mayores, a pensar que la Dirección atribuye más importancia a la información/asesoramiento.

Nos centramos a continuación en las diferencias significativas según el número de alumnado que atiende el Departamento de Orientación.

Los orientadores que atienden a centros pequeños, de menos de trescientos alumnos, tienden a pensar que el alumnado da más importancia al diagnóstico que los de los Centros medianos que tienen entre 301-700 alumnos. Este dato es coherente, en la medida en que un alumno, una familia, un profesor o un orientador, lógicamente pensará que atenderá más individualmente las dificultades que existan en la medida en que haya menos alumnado en el Centro. 
Tabla 12. Diferencias significativas en la importancia de las funciones del orientador según el número de alumnado que atiende el Departamento de Orientación.

\begin{tabular}{|c|c|c|c|c|c|}
\hline \multirow[b]{2}{*}{ Importancia de las funciones } & \multicolumn{3}{|c|}{ № Alumnos que atiende el D.O. } & \multirow[b]{2}{*}{$\mathbf{F}$} & \multirow[b]{2}{*}{ Sig. } \\
\hline & $\begin{array}{c}\text { Pequeño } \\
\text { (Menos de 300) }\end{array}$ & $\begin{array}{c}\text { Mediano } \\
(301-700)\end{array}$ & $\begin{array}{c}\text { Grande (Más } \\
\text { de } 700 \text { ) }\end{array}$ & & \\
\hline Diagnóstico según el alumnado & 3,61 & 3,24 & 3,14 & 3,690 & 0,028 \\
\hline
\end{tabular}

\begin{tabular}{|c|c|c|c|c|c|c|c|c|}
\hline \multirow{2}{*}{ Variable dependiente } & \multicolumn{3}{|c|}{ Comparaciones múltiples } & \multirow{2}{*}{$\begin{array}{l}\text { Diferencia } \\
\text { de } \\
\text { medias } \\
(1-J)\end{array}$} & \multirow{2}{*}{$\begin{array}{l}\text { Error } \\
\text { típico }\end{array}$} & \multirow[b]{2}{*}{ Sig. } & \multicolumn{2}{|c|}{$\begin{array}{c}\text { Intervalo de } \\
\text { confianza al 95\% }\end{array}$} \\
\hline & & $\begin{array}{l}\text { (I) alumnado que } \\
\text { atiende el Dpto. } \\
\text { de Orientación }\end{array}$ & $\begin{array}{l}\text { (J) alumnado que } \\
\text { atiende el Dpto. } \\
\text { de Orientación }\end{array}$ & & & & $\begin{array}{l}\text { Límite } \\
\text { inferior }\end{array}$ & $\begin{array}{l}\text { Límite } \\
\text { superior }\end{array}$ \\
\hline \multirow[t]{6}{*}{$\begin{array}{l}\text { Importancia del diagnóstico } \\
\text { según alumnado }\end{array}$} & \multirow[t]{6}{*}{ Scheffé } & \multirow[t]{2}{*}{$\begin{array}{l}\text { Pequeño } \\
(<\text { de } 300)\end{array}$} & $\begin{array}{l}\text { Mediano } \\
(301-700)\end{array}$ & 0,560 & 0,211 & 0,032 & 0,038 & 1,081 \\
\hline & & & $\begin{array}{c}\text { Grande } \\
\text { (> de 700) }\end{array}$ & 0,372 & 0,273 & 0,397 & $-0,303$ & 1,048 \\
\hline & & \multirow[t]{2}{*}{$\begin{array}{l}\text { Mediano } \\
(301-700)\end{array}$} & $\begin{array}{l}\text { Pequeño } \\
\text { (< de 300) }\end{array}$ & $-0,560$ & & 0,032 & $-1,081$ & $-0,038$ \\
\hline & & & $\begin{array}{c}\text { Grande } \\
\text { (> de 700) }\end{array}$ & $-0,187$ & 0,288 & 0,809 & $-0,900$ & 0,525 \\
\hline & & \multirow[t]{2}{*}{$\begin{array}{c}\text { Grande } \\
\text { (> de 700) }\end{array}$} & $\begin{array}{l}\text { Pequeño } \\
\text { (< de 300) }\end{array}$ & $-0,372$ & 0,273 & 0,397 & $-1,048$ & 0,303 \\
\hline & & & $\begin{array}{l}\text { Mediano } \\
(301-700)\end{array}$ & 0,187 & 0,288 & 0,809 & $-0,525$ & 0,900 \\
\hline
\end{tabular}

\section{CONCLUSIONES}

Las principales conclusiones obtenidas respecto al trabajo profesional de los orientadores y su percepción de la comunidad educativa son las siguientes.

En primer lugar, los orientadores perciben que las familias dan más importancia a las funciones referidas a la orientación académica y profesional -plena coincidencia en este aspecto con la percepción sobre el alumnado-, al diagnóstico y a la información. En este sentido, son congruentes los datos en la medida en que las familias se preocupan por el futuro profesional de sus hijos y por si tienen alguna dificultad específica para los estudios. Llama la atención cómo, alrededor de uno de cada cuatro orientadores, piensa que las familias no dan casi ninguna importancia al plan de acción tutorial. Dato preocupante y que puede ser revelador de las dificultades de comunicación o de las expectativas de los propios centros educativos y de las familias.

En segundo lugar, respecto a la percepción sobre la opinión que tiene el profesorado, se obtienen datos congruentes en todos los ítems en relación con los obtenidos por parte de las percepciones sobre los tutores. Sin embargo, todos ellos manifiestan índices más bajos, lo cual nos puede indicar el mayor grado de compromiso y relación que mantiene la red de tutores con los orientadores. El plan de atención a la diversidad, la orientación académica y profesional y el diagnóstico es lo que los orien- 
tadores piensan que dan más importancia, tanto el profesorado como los tutores. En relación a la Dirección, hay coincidencia respecto a estas funciones y los orientadores perciben que ésta lo que menos valora es la evaluación, innovación e investigación y la formación, reflejando de esta forma las relaciones dificultosas que, en ocasiones, se evidencian entre el propio Departamento de Orientación y la Dirección. Nos parece relevante señalar cómo una de las futuras líneas de trabajo se centra en potenciar la dimensión colaborativa entre estos estamentos. Uno de los objetivos sería disminuir las disonancias y la falta de coordinación que pudiera existir para implementar los diferentes programas de orientación en el proceso educativo.

Por último, los orientadores manifiestan de una forma clara, cómo sus percepciones, referidas a la Administración, reflejan las estimaciones más bajas en todas las funciones, de todos los colectivos a los que hace referencia la presente investigación. Datos preocupantes para mejorar, son los que afirman que cuatro de cada diez orientadores piensa que la Administración da poca o ninguna importancia a la formación de la comunidad educativa y casi la mitad lo expresa igualmente acerca de la evaluación, innovación e investigación, lo cual nos pone de manifiesto futuras líneas de trabajo para la propia Administración.

La perspectiva sistémica, y de trabajo colaborativo con necesidad de consensuar los diferentes objetivos educativos, priorizándolos, son algunos de los elementos que hay que potenciar en el ámbito de la orientación, y, específicamente de los orientadores en su relación con los demás miembros de la comunidad educativa.

Entre las limitaciones del estudio, que puede al mismo tiempo servir para futuras líneas de investigación, se encuentra la necesidad de incorporar muestras de cada uno de los miembros de la comunidad educativa, para contrastar dichos datos con las propias percepciones de los orientadores. La combinación con una metodología cualitativa, analizando información en grupos de discusión, por ejemplo, pueden aportar conclusiones más enriquecedoras.

Finalmente hemos de concluir que la gran dispersión de dicho trabajo profesional por parte de estos profesionales, evidencia claramente una intervención sobre casos problemáticos -a veces desde una perspectiva demasiado clínica-, más que desde un enfoque preventivo. Finalmente, sobresalen algunas funciones como la formación o la evaluación, innovación e investigación, que no tienen el papel clave que deberían desempeñar, en nuestra opinión, para la mejora de la calidad de la educación.

\section{REFERENCIAS BIBLIOGRÁFICAS}

ALANIZ, M. (1990): High school teachers and guidance counselors: Differents roles, common goals. Social Studies Review: Journal of the California Council for the Social Studies 30, 49-61.

CANO, J. (2006): Análisis de las funciones de los orientadores en la Educación Secundaria Obligatoria en la Comunidad Autónoma de Aragón. Madrid: Universidad Complutense. Tesis doctoral.

FUENTES, L. M. y LLEDÓ, A. (2007): El asesoramiento al profesorado y a las familias en la orientación educativa. En LLEDÓ, A. (Coord.) (2007): La orientación educativa desde la práctica. Sevilla: Fundación ECOEM. 
GARCÍA NIETO, N. et al. (2005): La tutoría Universitaria ante el proceso de Armonización Europea. Revista de Educación 337, 189-210.

GARCÍA NIETO, N. y CANO, J. (2008): Aproximación al perfil del orientador en la ESO de Aragón. REOP 19, 3, 303-315.

GIBSON, R. L. (1990): Teachers opinions of high school counseling and guidance programs: Then and now, School Counselor 37, 248-255.

GONZÁLEZ, I. et al. (2007): Elaboración de un perfil de la orientación en Educación Secundaria desde las demandas de la comunidad educativa. REOP 18, 2, 215-227.

HUTCHINSON, R. L. et al. (1986): Functions of secondary school counselors in the public schools: Ideal and actual. School Counselor 43, 2, 87-91.

HUTCHINSON, R. L. (1989): Problems for which seniors would seek help from school counselors. School Counselor 33, 350-354.

LLEDÓ, A. (Coord.) (2007): La orientación educativa desde la práctica. Sevilla: Fundación ECOEM.

ROWE, F. A. (1989): College students' perceptions of high school counselors. School Counselor 36, 260-264.

SANZ, R. (1999): Los Departamentos de Orientación en Educación Secundaria: Roles y funciones. Barcelona: Cedecs.

VALINE, W. J. et al. (1982): Teacher attitudes toward the role of the coanselor: An eight year follow-up study. School Counselor 29, 208-211.

WELLS, C. E. et al. (1979): Paperwork, pressure and discouragement: Student attitudes toward guidance services and implications for the profession. Personnel and Guidance Journal 58, 170-175. 